\title{
Complete Elimination of DC Offset in Current Signals for Relaying Applications
}

\author{
Jun-Zhe Yang \\ Chih-Wen Liu \\ Department of Electrical Engineering, National Taiwan University, Taipei, Taiwan
}

\begin{abstract}
This paper proposes a new method for exact estimation of DC offset in current signals such that DC offset can be completely eliminated for relaying applications. This new method, we called Smart Discrete Fourier Transform (SDFT), based on Discrete Fourier Transform (DFT) keeps all advantages of DFT, and smartly avoids the defects resulting from that frequency scales can't match signal characteristics. Moreover, we use smoothing windows to filter noise in practice. The difference between SDFT and other methods, which use smoothing windows, is that SDFT can alleviate the difficulties of phase shift and amplitude decay caused by smoothing windows. Recursive and half-cycle computing can also be used in SDFT, so SDFT is very suitable for use in real-time. To validate the claimed benefits of SDFT, the performances of SDFT algorithm are demonstrated with the data generated by EMTP simulator. The simulation results are very encouraging.
\end{abstract}

Keywords: Discrete Fourier Transforms (DFT), Frequency estimation, phasor measurement.

\section{Introduction}

In power systems, high voltage transmission and distribution lines are vital links from the generating plants to the end users. From views of security, economics, and quality of power feeding, it is necessary for protecting lines using relay [1]. When fault occurs, relays prevent involving area from growing. Hence correct action of relay is important in power systems. However, it is a fact that a line relay tends to overreach in the presence of DC offset component in fault current waveforms. Therefore, DC offset component has to be removed from these waveforms $[2,3]$.

SDFT can estimate DC offset component exactly. In fact, SDFT obtains exact DC offset from fault current waveforms such that it can be eliminated. This is useful for fault detection and location. Three advantages of using SDFT are presented here. First, relays can work correctly. Without influence of DC offset, relays obtain accurate frequency and phasor components from SDFT. Second, $L / R$ time constant of $D C$ offset can be used in line parameter estimation after fault occurs. Uncertainty about the line parameters, which usually results in a significant error in the calculated fault location, can also be resolved by SDFT. Third, SDFT obtains exact fault angle of DC offset. Therefore, one can use this extra information in protection and control.
Noise is unavoidable in power systems. Hence every algorithm applied to power systems has to take noise into consideration. Currently more prevalent methods are using smoothing windows. Generally, using smoothing windows is known to provide flexible solutions to filtering noise, but loses the ability of computing phasors. SDFT can allay the phase shift and amplitude decay programs caused by smoothing windows.

This paper is organized into four sections, the first of which is introduction. In section II, we introduce the proposed algorithm, SDFT, used in estimating DC offset, and prove that SDFT can obtain phasors when using smoothing windows. We test SDFT using EMTP simulation data in Section III. Finally, section IV is devoted to conclusions.

\section{The Proposed Digital Algorithm}

This section proposes the algorithm of SDFT that estimates the DC offset, frequency, and phasor from a fault current signal. Since there are several components in a fault current signal [4-6], the algorithm first takes DC offset into consideration and uses smoothing windows to eliminate other components in a fault current signal. The main components of a fault current signal can be expressed as follows [7]:

$$
x(t)=X \sin \left(\omega t+\phi_{1}\right)+X \sin \left(\phi_{2}\right) \exp (-\alpha t)
$$

where $X:$ the amplitude of the signal, $\phi_{1}$ : the phase angle of the signal, $\phi_{2}$ : the fault angle of the signal, $\frac{1}{\alpha}=\tau:$ the time constant of the signal

Suppose that $x(t)$ is sampled with a sampling rate $(60 * \mathrm{~N}) \mathrm{Hz}$ to produce the sample set $\{x(k)\}$.

$$
x(k)=X \sin \left(\omega \frac{k}{60 N}+\phi_{1}\right)+X \sin \left(\phi_{2}\right) \exp \left(-\alpha \frac{k}{60 N}\right)
$$

The signal $x(t)$ is conventionally represented by a phasor (a complex number) $\bar{x}$.

$$
\bar{x}=X e^{\phi_{1}}=X \cos \phi_{1}+j X \sin \phi_{1}
$$

Then $x(t)$ can be expressed as

$$
x(t)=\frac{\bar{x} e^{\mu t}-\tilde{x}^{*} e^{-\mu+t}}{2}+X \sin \left(\phi_{2}\right) \exp \left(-\alpha \frac{k}{60 N}\right)
$$


where * denotes complex conjugate.

Moreover, the fundamental frequency $(60 \mathrm{~Hz})$ component of DFT of $\{x(k)\}$ is obtained by

$$
\hat{x}_{r}=\frac{2}{N} \sum_{k=0}^{N-1} x(k+r) e^{-\frac{2 \pi k}{N}}
$$

Combing Eq. (4) and Eq. (5) and taking frequency deviation $(\omega=2 \pi(60+\Delta f))$ into consideration, we obtain

$$
\begin{aligned}
\hat{x}_{r} & =\frac{\bar{x}}{N} \sum_{k=0}^{N-1} e^{j 2 \pi(60+\Delta) \frac{(k+r)}{60 N}} e^{-j 2 \pi \frac{k}{N}} \\
& -\frac{\bar{x}}{N} \sum_{k=0}^{N-1} e^{-j 2 \pi(60+\Delta) \frac{(k+r)}{60 N}} e^{-j 2 \pi \frac{k}{N}} \\
& +\frac{2 X \sin \phi_{2}}{N} \sum_{k=0}^{N-1} e^{-\alpha \frac{k+r}{60 N}} e^{-j 2 \pi \frac{k}{N}}
\end{aligned}
$$

We rearrange Eq. (6) as the following:

$$
\begin{aligned}
\hat{x}_{r} & =\frac{\bar{x}}{N} e^{j \frac{2 \pi}{N}\left(1+\frac{\mathcal{N}}{60}\right) r} \sum_{k=0}^{N-1} e^{j 2 \pi \frac{\alpha}{60 N} k} \\
& -\frac{\bar{x}^{*}}{N} e^{-j \frac{2 \pi}{N}\left(1+\frac{\psi}{60}\right) r} \sum_{k=0}^{N-1} e^{-j 2 \pi \frac{\left(2+\frac{\psi}{60}\right)}{N} k} \\
& +\frac{2 X \sin \phi_{2}}{N} e^{-\alpha \frac{r}{60 N}} \sum_{k=0}^{N-2} e^{-\pi \frac{k}{60 N}} e^{-j 2 \pi \frac{k}{N}}
\end{aligned}
$$

We use the following identity to simplify Eq. (7).

$$
\sum_{i=0}^{N-1}\left(e^{e}\right)^{\prime}=\frac{\sin \frac{N \theta}{2}}{\sin \frac{\theta}{2}} e^{(N-1) \frac{\theta}{2}}
$$

Then Eq. (7) can be expressed as

$$
\begin{aligned}
\hat{x}_{r} & =\frac{\bar{x}}{N} e^{j \frac{2 \pi}{N}\left(1+\frac{\Delta f}{60}\right) r} \frac{\sin N \theta_{1}}{\sin \theta_{1}} e^{J(N-1) \theta_{1}} \\
& -\frac{\bar{x}}{N} e^{-\frac{2 \pi}{N}\left(1+\frac{\Delta f}{60}\right) r} \frac{\sin N \theta_{2}}{\sin \theta_{2}} e^{J(N-1) \theta_{2}} \\
& +\frac{2 X \sin \phi_{2}}{N} \frac{e^{-\frac{a}{60}}-1}{e^{-\frac{a}{60 N}-j \frac{2 \pi}{N}}-1} e^{-\frac{a r}{60 N}}
\end{aligned}
$$

Where $\theta_{1}=\frac{\pi \Delta f}{60 N}$, and $\theta_{2}=-\frac{\pi\left(2+\frac{\Delta f}{60}\right)}{N}$.

Rearranging Eq. (9) further, we obtain

$$
\begin{aligned}
\hat{x}_{r}^{(60+N)}= & \frac{\bar{x}}{N} \frac{\sin N \theta_{1}}{\sin \theta_{1}} e^{j \frac{\pi}{60 N}(A N(2 r+N-1)+120 r)} \\
& -\frac{\bar{x} \cdot}{N} \frac{\sin N \theta_{2}}{\sin \theta_{2}} e^{\left.-\frac{\pi}{60}(N)(2 r+N-1)+120(r+N-1)\right)} \\
& +\frac{2 X \sin \phi_{2}}{N} \frac{e^{-\frac{a}{60}}-1}{e^{-\frac{a}{60 N}-i \frac{2 \pi}{N}}-1} e^{-\frac{a r}{60 N}}
\end{aligned}
$$

We define $A_{r}, B_{r}$ and $C_{r}$ as follows:

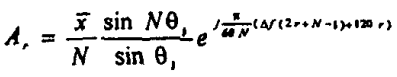

$$
\begin{aligned}
& B_{r}=-\frac{\bar{x}^{*}}{N} \frac{\sin N \theta_{3}}{\sin \theta_{2}} e^{-\frac{\pi}{\omega 0 N}(\Delta /(2 r+N-1)+120(r+N-1))}
\end{aligned}
$$

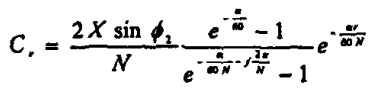

Then Eq. (10) can be expressed as

$$
\hat{x}_{r}=A_{r}+B_{r}+C_{r}
$$

So far the development of the algorithm of SDFT is the same as the traditional DFT method. So the SDFT can keep all advantages of DFT such as recursive and half-cycle computing manner. But in the DFT, it doesn't take DC offset into consideration and it assumes that the frequency deviation is small enough to be ignored. It always considers $\hat{x}_{r} \approx A_{r}$, so traditional DFT based methods incur error in estimating frequency and phasor when frequency deviates from nominal frequency $(60 \mathrm{~Hz})$ or DC offset is present. If we want to obtain exact solution, we must take $B_{r}$ and $C_{r}$ into consideration. Then we define

$$
a=e^{j\left(\frac{x}{60 N}(24+120)\right)}
$$

and

$$
b=e^{\frac{-\alpha}{60 N}}
$$

And from Eq. (10), we will find the following relations.

$$
\begin{aligned}
& A_{r+1}=A_{r} a \\
& B_{r+1}=B_{r} a^{-1} \\
& C_{r+1}=C_{r} b
\end{aligned}
$$

Then

$\hat{x}_{r+1}=A_{r+1}+B_{r+1}+C_{r+1}=A_{r} a+B_{r} a^{-1}+C_{r} b$
$\hat{x}_{r+2}=A_{r+2}+B_{r+2}+C_{r+2}=A_{r+1} a+B_{r+1} a^{-1}+C_{r+1} b$

Multiplying both sides of Eq. (14) with ' $b$ ' and 
subtracting from Eq. (20) give

$$
\hat{y}_{r}=\hat{x}_{r+1}-\hat{x}_{r} b=A_{r}(a-b)+B_{r}\left(a^{-1}-b\right)
$$

Similarly, we may obtain

$$
\begin{aligned}
& \hat{y}_{r+1}=\hat{x}_{r+2}-\hat{x}_{r+1} b=A_{r+1}(a-b)+B_{r+1}\left(a^{-1}-b\right) \\
& \hat{y}_{r+2}=\hat{x}_{r+3}-\hat{x}_{r+2} b=A_{r+2}(a-b)+B_{r+2}\left(a^{-1}-b\right)
\end{aligned}
$$

As same treatment with Eq. (14) and Eq. (20), we may rearrange Eq. (22), Eq. (23), and Eq. (24) to obtain

$$
\begin{aligned}
& \hat{y}_{r+1} a-\hat{y}_{r}=A_{r}\left(a^{2}-1\right)(a-b) \\
& \hat{y}_{r+2} a-\hat{y}_{r+1}=A_{r+1}\left(a^{2}-1\right)(a-b)
\end{aligned}
$$

Dividing Eq. (25) by Eq. (26), we get

$$
a=\frac{A_{r+1}}{A_{r}}=\frac{\hat{y}_{r+2} a-\hat{y}_{r+1}}{\hat{y}_{r+1} a-\hat{y}_{r}}=\frac{\hat{y}_{r+3} a-\hat{y}_{r+2}}{\hat{y}_{r+2} a-\hat{y}_{r+1}}
$$

Then substituting Eq. (23) and Eq. (24) into Eq. (27) and eliminating variable ' $a$ ', we obtain as follows:

$$
\begin{aligned}
& {\left[\hat{x}_{r+2}\left(\hat{x}_{r}+\hat{x}_{r+2}\right)-\hat{x}_{r+1}\left(\hat{x}_{r+1}+\hat{x}_{r+3}\right)\right] b^{2} } \\
+ & {\left[\hat{x}_{r+3}\left(\hat{x}_{r}+\hat{x}_{r+2}\right)-\hat{x}_{r+1}\left(\hat{x}_{r+2}+\hat{x}_{r+1}\right) b\right.} \\
+ & {\left[\hat{x}_{r+3}\left(\hat{x}_{r+1}+\hat{x}_{r+3}\right)-\hat{x}_{r+2}\left(\hat{x}_{r+2}+\hat{x}_{r+4}\right)\right]=0 }
\end{aligned}
$$

Solve Eq. (28) to obtain ' $b$ '. From the definition of ' $b$ ' in Eq. (16), we can obtain the exact solution of the time constant.

$$
\tau=\frac{1}{60 N \log b}
$$

Then rearrange Eq. (27) as follows

$$
\hat{y}_{r+1} a^{2}-\left(\hat{y}_{r}+\hat{y}_{r+2}\right) a+\hat{y}_{r+1}=0
$$

Solve Eq. (30) to obtain ' $a$ '. From the definition of ' $a$ ' in Eq. (16), we can get the exact solution of the frequency.

$$
f=60+\Delta f=\cos ^{-1}(\operatorname{Re}(a)) \frac{60 N}{2 \pi}
$$

From Eq. (29) and Eq. (31), it is observed that SDFT can provide exact time constant and frequency using $\hat{x}_{r}, \hat{x}_{r+1}$, $\hat{x}_{r+2}, \hat{x}_{r+3}$, and $\hat{x}_{r+4}$ in the absence of noise. Moreover, we can estimate phasor and fault angle after getting exact time constant and frequency by the following equations:

$$
\begin{aligned}
& A_{r}=\frac{\hat{y}_{r+1} a-\hat{y}_{r}}{\left(a^{2}-1\right)(a-b)} \\
& X=a b s\left(A_{r} \frac{N \sin \left(\theta_{1}\right)}{\sin \left(\mathrm{N} \theta_{1}\right)}\right) \\
& \phi_{1}=\operatorname{angle}\left(A_{r} e^{-j \theta_{1}(N-1)}\right) \\
& C_{r}=\frac{a^{2} \hat{x}_{r+1}-a\left(\hat{x}_{r}+\hat{x}_{r+2}\right)+\hat{x}_{r+1}}{(a-b)(a b-1)} \\
& \phi_{2}=\sin ^{-3}\left(\frac{C_{r} N}{2 X} \frac{e^{-\frac{\alpha}{60 N}-j \frac{2 \pi}{N}}-1}{e^{-\frac{a}{6 \theta}}-1}\right)
\end{aligned}
$$

Furthermore, we take noise into consideration and use smoothing windows to filter noise. Consider a sampled set $\{x(k)\}$ to be a filtered set $\{z(k)\}$ by a smoothing window $\left\{S W(m) \mid s_{1}, s_{2}, \cdots s_{s_{m}}\right\}, \mathrm{m}$ is window size.

$$
z(k)=\sum_{i=1}^{m} s_{i} x(k+i-1)
$$

Moreover, the DFT of $\{z(k)\}$ is given by

$$
\begin{aligned}
\hat{z}_{r} & =\frac{2}{N} \sum_{k=0}^{N-1} z(k+r) e^{-j \frac{2 \pi t}{N}} \\
& =\frac{2}{N} \sum_{k=0}^{N-1}\left[\sum_{i=1}^{m} s_{i} x(k+r+i-1)\right] e^{-j \frac{2 \pi t}{N}} \\
& =\sum_{i=1}^{m} s_{i}\left[\frac{2}{N} \sum_{k=0}^{N-1} x(k+r+i-1) e^{-j \frac{2 \pi k}{N}}\right] \\
& =\sum_{i=1}^{m} s_{i} \hat{x}_{r+1-1}
\end{aligned}
$$

From the definition of Eq. (14), we can obtain:

$$
\begin{aligned}
\hat{z}_{r} & =A_{1}\left(s_{1}+s_{2} a+\cdots+s_{m} d^{m-1}\right) \\
& +B_{r}\left(s_{1}+s_{2} a^{-1}+\cdots+s_{m} a^{-m+1}\right) \\
& +C_{r}\left(s_{1}+s_{2} b+\cdots+s_{m} b^{m-1}\right)
\end{aligned}
$$

The relations of Eq. (17), Eq. (18), and Eq. (19) are still kept in Eq. (39). Therefore, the same steps from Eq. (20) to Eq. (33) can be used in Eq. (39). Hence we can estimate time constant and frequency without modifying equations, but we have to do some change in Eq. (32) and Eq. (35) when we estimate phasor and fault angle.

$$
A_{r}=\frac{\hat{y}_{r+1} a-\hat{y}_{r}}{\left(a^{2}-1\right)(a-b)\left(s_{1}+s_{2} a+\cdots+s_{-} a^{-1-1}\right)}
$$

where $\hat{y}_{r}=\hat{z}_{r+1}-\hat{z}_{r} b$ and $\hat{y}_{r+1}=\hat{z}_{r+2}-\hat{z}_{r+1} b$ 


$$
C_{r}=\frac{a^{2} \hat{x}_{r+1}-a\left(\hat{x}_{r}+\hat{x}_{r+2}\right)+\hat{x}_{r+1}}{(a-b)(a b-1)\left(s_{1}+s_{2} b+\cdots+s_{m} b^{m-1}\right)}
$$

The phasor obtained from Eq. (40) and fault angle obtained from Eq. (41) will allay the phase shift and amplitude decay caused by smoothing windows. In the next section, we will test the SDFT method with the data generated by EMTP simulator.

\section{Simulation Results}

The first example of EMTP simulation is a single-phase system and the model is shown Fig. I. It is a $100 \mathrm{~km}$ transmission line connected to generators at sending and receiving end, respectively. The transmission line model used in the simulation is the distributed parameters model.

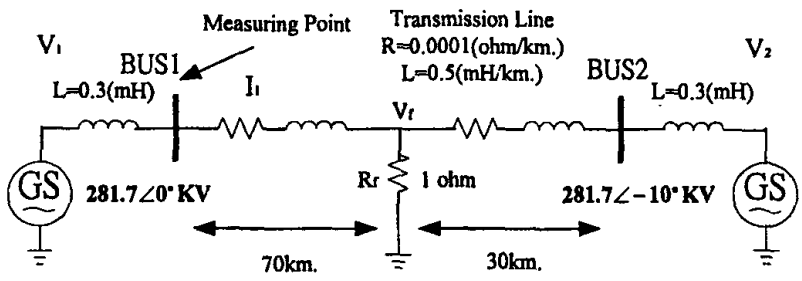

Fig.1 EMTP simulation model $(f=60 \mathrm{~Hz})$

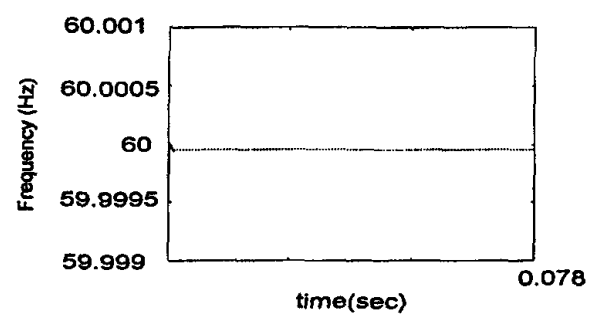

Fig. 2(a)

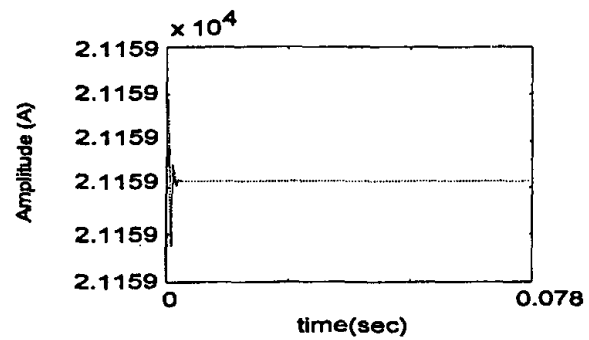

Fig. 2(c)

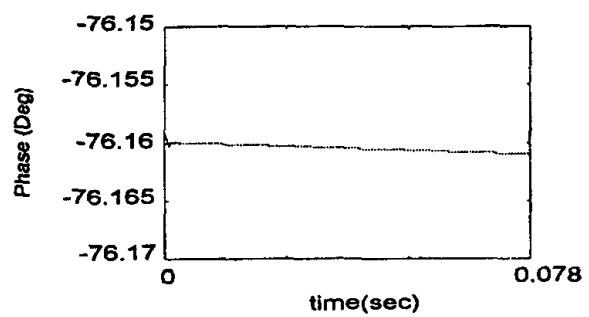

Fig. 2(c)
A fault is simulated to occur at the peak value of voltage $V_{1}$ and we measured current $I_{1}$ at BUS1. Computation results by the SDFT are showed in Fig. 2 and theoretical results are as follows:

$$
\begin{aligned}
\tau & =\frac{\left(0.0003+0.0005^{*} 70\right) \|(0.0003+0.0005 * 30)}{(0.0001 * 70) \|(0.0001 * 30)+1} \\
& =0.01065135=10.65135 * 10^{-3} \\
z 1 & =0.0001 * 70+j \omega^{*}(0.0003+0.0005 * 70) \\
z 2 & =0.0001 * 30+j \omega^{*}(0.0003+0.0005 * 30) \\
V_{f} & =\frac{z 1 * V_{2}+z 2 * V_{1}}{z 1+z 2+z 1 * z 2}=67714 \angle-82.9962^{*}
\end{aligned}
$$

The current of measurement point is

$$
I_{1}=\frac{V_{1}-V_{1}}{z 1}=21159 \angle-76.1611^{\circ}
$$

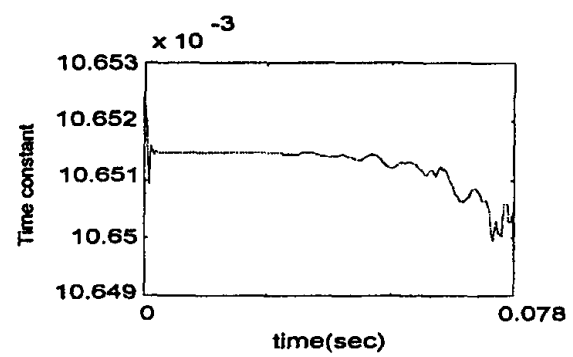

Fig. 2(b)

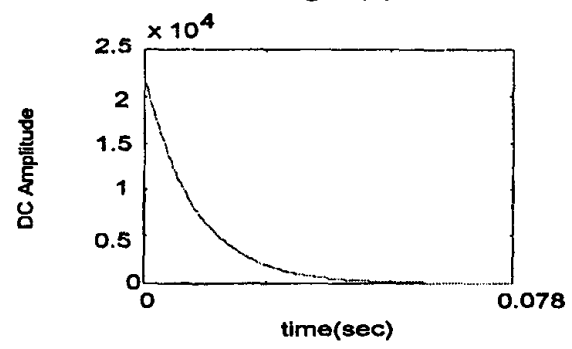

Fig. 2(d)

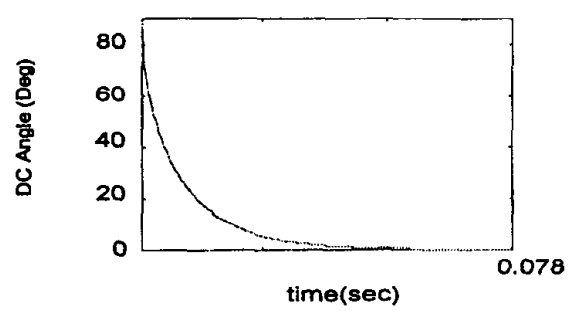

Fig. 2(f)

Fig. 2 Computation results by SDFTds 
To distinguish different kind of SDFT [8], we add ' $d$ ' and ' $s$ ' in the suffix of SDFT means this kind of SDFT has taken DC offset and Smoothing windows into consideration. In this example, we used Blackman window and window size is 28 points and SDFTd moving window size is 36 points. It means SDFTds gets first computing results after fault occurred 2 cycles. From Fig.2, it is clear that SDFTds succeed in eliminating DC offset. Since the amplitude of DC offset is decaying with time, the computing result of time constant will become senseless.

Next example, we add capacitance into model, change system frequency and change location and time of a fault. Computation results are showed in Fig. 4 and theoretical results are as follows:

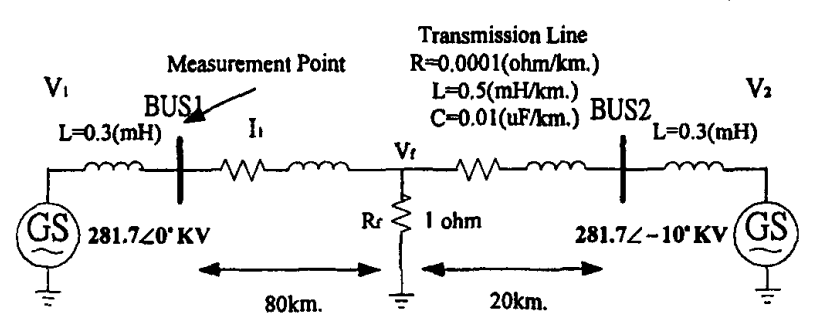

Fig.3. EMTP simulation model $(f=60.5 \mathrm{~Hz})$

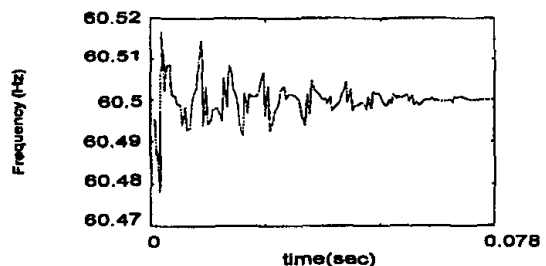

Fig. 4(a)

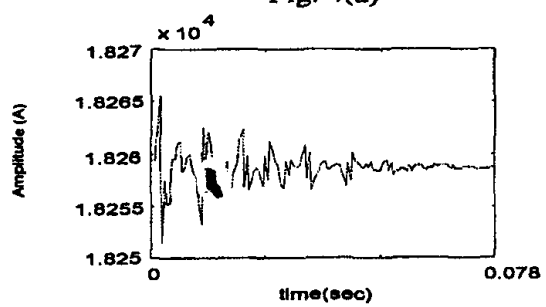

Fig. 4(c)

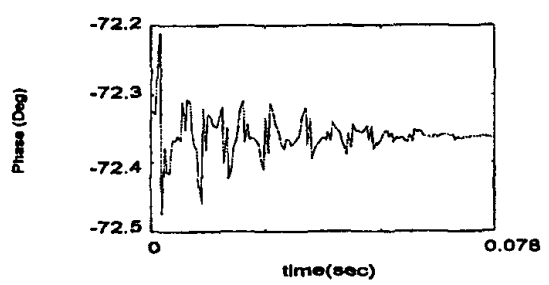

Fig. 4(e)

$$
\begin{aligned}
& \tau=\frac{(0.0003+0.0005 * 80) \|(0.0003+0.0005 * 20)}{(0.0001 * 80) \|(0.0001 * 20)+1} \\
&=0.00819025=8.19025 * 10^{-3} \\
& Z s=\left[\begin{array}{cc}
1 & j \omega 3 e^{-4} \\
0 & 1
\end{array}\right] \quad L s=\left[\begin{array}{cc}
1 & 1 e^{-4}+j \omega 5 e^{-4} \\
0 & 1
\end{array}\right] \\
& L p=\left[\begin{array}{cc}
1 & 0 \\
j \omega 1 e^{-s} & 1
\end{array}\right] \quad Z f=\left[\begin{array}{ll}
1 & 0 \\
1 & 1
\end{array}\right] \\
& {\left[\begin{array}{cc}
A & B \\
C & D
\end{array}\right]=Z s *(L s * L p)^{80} * Z f^{*}(L s * L p)^{20} * Z s }
\end{aligned}
$$$$
\left.\left[\begin{array}{l}
I_{1} \\
I_{2}
\end{array}\right]=\left[\begin{array}{cc}
\frac{D}{B} & C-\frac{D A}{B} \\
\frac{1}{B} & -\frac{A}{B}
\end{array}\right] \begin{array}{l}
V_{1} \\
V_{2}
\end{array}\right]
$$$$
\left.=\left[\begin{array}{cc}
0.039-0.0639 i & 0.0151+0.0049 i \\
-0.0151-0.0049 i & -0.0593+0.2364 i
\end{array}\right] \begin{array}{c}
281.7 * 10^{3} \\
281.7 * 10^{3} \angle-10^{\circ}
\end{array}\right]
$$$$
I_{1}=18259 \angle-72.3607^{\circ} \text { (A) }
$$

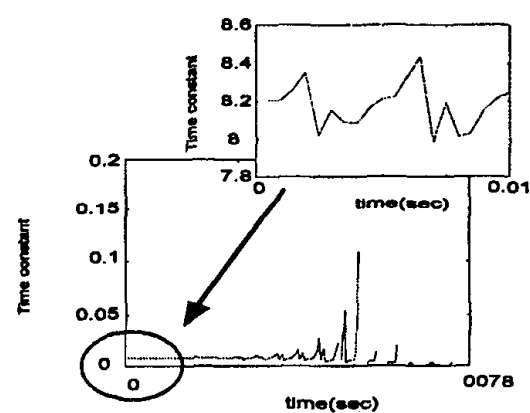

Fig. 4(b)

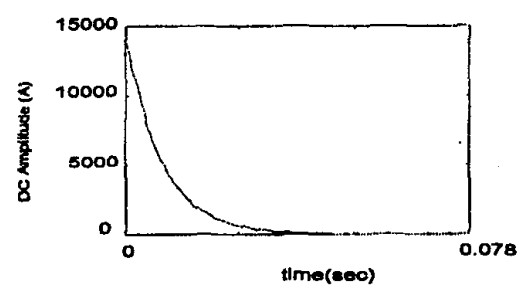

Fig. 4(d)

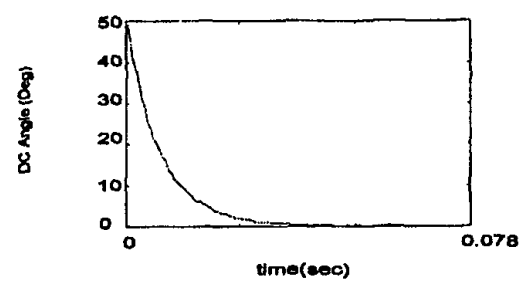

Fig. 4(f)

Fig. 4. Computation results by SDFTds 
Since we added capacitance into the model, the transient current signal added high frequency component that caused by resonance between line capacitance and line inductance [4-6]. In this example, the high frequency component of transient current signal doesn't make serious affection to computing results of SDFTds. Actually, added window size of smoothing windows is helpful to resist the high frequency component and noise of transient current signal, but how large of window size can be added depends on how long the line breaker open. Generally speaking, the fault will be cleared after 3 cycles, and it should be enough time for using SDFTds in fault location or other applications.

\section{Conclusion}

In this paper we introduce the SDFT method and demonstrate its performance with the data generated by EMTP simulator. From the Examples presented above, SDFT succeed in eliminating DC offset. SDFT both keeps the advantages of DFT and also deals with the cause of DC offset errors, while taking smoothing windows into consideration. These aspects make SDFT an accurate and practical method in line relaying applications.

\section{Reference}

[1] J. A. Jiang, Y. H. Lin, C. W Liu, J. Z Yang, T. M. Too, "An Adaptuve Fault Locator System For Transmission Lines," JEEE/PES Summer Meeting, July 1999, pp. 930-936

[2] J. C. Gu and S. L. Yu, "Removal of DC Offset in Current and Voltage Signals Using a Novel Fourier Filter Algorithm," IEEE Trans. on Power Deltvery, PE-052PRD(06-99).

[3] Gabriel Benmouyal, "Removal of DC-Offset in Current Waveforms Using Digital Mimic Filtering," IEEE Trans on Power Deltvery. Vol. 10, No. 2, April 1995, pp. 621-630.

[4] Adly A. Girgis, A. A. Sallam, A. Karim El-Din, "An Adaptive Protection Scheme for Advanced Series Compensated (ASC) transmission Lines," IEEE Trans. on Power Delivery, Vol. 13, No. 2, April 1998, pp. 414-420.

[5] Adly A. Girgis and R. Grover Brown, "Modeling of Fault-Induced Noise Signals for Computer relaying Applications," IEEE Trans. on Power Apparatus and Systems, Vol. PAS-102, No. 9, September 1983, pp 2834-2841.

[6] Adly A. Girgis and R. Grover Brown, "Application of Kalman Filtering in Computer Relaying," IEEE Trans. on Power Apparatus and Systems, Vol PAS-100, July 1981, pp. 3387-3397.

[7] Norman T. Stringer, "The Effect of DC Offset on CurrentOperated relays," IEEE Trans. on Industry Applications, Vol. 34, No. 1, January/February 1998, pp. 30-34.

[8] J. Z Yang and C. W. Liu, "A Smart Method Makes DFT More Precise for Power System Frequency Estimation," IEEE/PES Winter Meeting, Feb. 1999, pp. 909-913.

\section{Biographies}

hih-Wen Liu was born in Taiwan in 1964. He received the B.S. degree in Electrical Engineering from National Taiwan University in 1987, Ph.D. degree in electrical engineering from Cornell University in 1994. Since 1994, he has been with National Taiwan University, where he is associate professor of electrical engineering. His research interests include application of numerical methods to power system, motor control and GPS time transfer.

Jun-Zhe Yang was born at Tainan, Taiwan, in 1971. He received his B.S. degree in electrical engineering from Tatung Institute of Technology in 1992 and M.S. degree from National Taiwan University in 1995 . He is presently a graduate student in the electrical engineering department, National Taiwan University, Taipei, Taiwan. 